Commun. Fac. Sci. Univ. Ank. Ser. A1 Math. Stat.

Volume 69, Number 1, Pages 137-157(2020)

DOI: $10.31801 /$ cfsuasmas. 567496

ISSN 1303-5991 E-ISSN 2618-6470

http://communications.science.ankara.edu.tr/index.php?series=A1

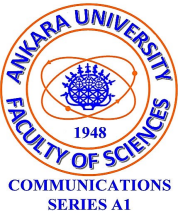

\title{
TRANSMUTED GUMBEL UNIVARIATE EXPONENTIAL DISTRIBUTION
}

\author{
MONIREH HAMELDARBANDI AND MEHMET YILMAZ
}

\begin{abstract}
A functional composition of the distribution function of one probability distribution with the inverse distribution function of another is called the transmutation map. The present paper is purported to show how the transmuted distribution can be obtained by using the convex combination of failure probability of two-component systems. The transmuted Gumbel univariate exponential distribution is presented by changing convex combination parameter. This new distribution is defined and studied. Some mathematical properties of this distribution including the generating function and ordinary moments are derived. The survival, hazard rate and mean residual life functions are discussed. Finally, three applications to real data are presented.
\end{abstract}

\section{INTRODUCTION}

In the present paper, we will start by examining two-component (series and parallel) systems. The failure probabilities of these systems will be found and a new distribution is obtained by applying convex combinations to these probabilities as these can be ordered within themselves. In the process of proposing this distribution, the lifetimes of the components of the system which are the random variables are considered to be both dependent on each other and non-identical. If the random variables that represent the lifetimes of two components are identical and independent, then this proposed distribution will emerge in the transmuted model, which is one of the important families in the pertinent-literature in recent years. The transmuted family has been introduced by [27] for the first time and the theory of transmuted distribution is clearly defined by 28. This method has led to the development of new and more flexible distributions by many authors, proposing many different distributions and pioneering the modeling of many real data sets with these distributions. Aryal and Tsokos [4] and [5] studied the two forms of

Received by the editors: May 19, 2019; Accepted: September 02, 2019.

2010 Mathematics Subject Classification. Primary 05C38, 15A15; Secondary 05A15, 15A18.

Key words and phrases. Gumbel exponential distribution, convex combination, transmutation method, hazard rate function, exponential distribution.

(C)2020 Ankara University Communications Faculty of Sciences University of Ankara-Series A1 Mathematics and Statistics 
the transmuted distributions. These scholars provided the mathematical characterization of transmuted extreme value and transmuted Weibull distributions and their applications to analyze real data sets. Aryal [6] proposed the transmuted loglogistic distribution and discussed various properties of this distribution. Merovci [19] introduced the transmuted Lindley distribution and applied it to bladder cancer data; Merovci [20] proposed the transmuted exponentiated exponential distribution; Merovci and Elbatal 21] studied the transmuted Lindley-geometric distribution. Ashour and Eltehiwy [7] discussed the applications of Transmuted Lomax Distribution and Ashour and Eltehiwy 8 proposed the transmuted exponentiated Lomax distribution. More recently, the transmuted exponentiated modified Weibull distribution has been suggested by [13] having its applications in real data. Hussian [16] obtained the transmuted exponentiated gamma distribution and discussed their various properties and applications. Elbatal et al. 11] discussed as various estimation methods for the transmuted exponentiated Fréchet distribution. Abd El Hady [1] obtained an extended Weibull distribution as the exponentiated transmuted Weibull distribution and discussed its various properties and applications. Merovci and Puka 22] introduced the transmuted Pareto distribution. Elbatal and Aryal [12] studied the transmuted additive Weibull distribution; Merovci [23] proposed the transmuted Rayleigh distribution and discussed their various properties. In the second part of this article, the new family will be introduced and the survival and hazard rate functions of the model under study will be found. The third part of this article contains some main definitions as Gumbel Bivariate Exponential Distribution and Gumbel Univariate Exponential Distribution. Later, the baseline distributions of the proposed distribution will be taken as exponential distribution and the proposed distribution is called the transmuted Gumbel univariate exponential (TGUE) distribution. In the subsequent subsections, the analytical shapes of the probability density, survival, cumulative hazard rate, hazard rate and mean residual life functions of the TGUE distribution are presented. Statistical properties including moment generating function and moments, maximum likelihood estimates and the information matrix, random number generation, Rényi entropy and order statistics of the TGUE distribution are discussed in other subsections of Section 3. Finally, in order to demonstrate the usefulness of the proposed distribution, three real data applications are presented in the application section.

\section{The New Family}

In recent literature, the transmuted family of lifetime distributions have attracted the attention of the researchers for modeling the lifetime data. Firstly, two-component (series and parallel) systems will be introduced. Let $T_{1}$ and $T_{2}$ be random variables that represent the lifetime of the components. Throughout this paper, the marginal distribution functions of $T_{1}$ and $T_{2}$ are represented by $F_{T_{1}}$ (.) and $F_{T_{2}}($.$) , and the joint distribution and the joint survival functions of T_{1}$ and 
$T_{2}$ are indicated by $F_{T_{1}, T_{2}}(.,$.$) and S_{T_{1}, T_{2}}(.,)=.1-F_{T_{1}}()-.F_{T_{2}}()+.F_{T_{1}, T_{2}}(.,$.$) ,$ respectively. The series system success requires that the two parts operate successfully at the same time. System failure occurs if either one or more components fail. Then, the random variable $T_{m i n}$ that stands for the series system lifetime is defined as $T_{\min }=\min \left\{T_{1}, T_{2}\right\}$. Hence, the probability of the failure of the series system is given by

$P\left(T_{\min } \leq t\right)=1-P\left(T_{1}>t, T_{2}>t\right)=1-S_{T_{1}, T_{2}}(t, t)=F_{T_{1}}(t)+F_{T_{2}}(t)-F_{T_{1}, T_{2}}(t, t)$

Parallel system is such a system that functions when at least one of its components works and the failure of all the components is necessary for the system's failure to occur. Accordingly, $T_{\max }=\max \left\{T_{1}, T_{2}\right\}$ stands for the parallel system lifetime. Then, the probability of the failure of the parallel system is given by

$$
P\left(T_{\max } \leq t\right)=P\left(\max \left\{T_{1}, T_{2}\right\} \leq t\right)=P\left(T_{1} \leq t, T_{2} \leq t\right)=F_{T_{1}, T_{2}}(t, t) .
$$

According to axiomatic properties of probability, component lifetimes $T_{1}$ and $T_{2}$ can be ordered stochastically as $T_{\min } \leq{ }_{s t} T_{i} \leq{ }_{s t} T_{\max }, i=1,2$. Namely, we have $P\left(T_{\max } \leq t\right) \leq P\left(T_{i} \leq t\right) \leq P\left(T_{\min } \leq t\right)$. Then, the lower and the upper bounds for $F_{T_{i}}(t)$ can be written as follows:

$$
F_{T_{1}, T_{2}}(t, t) \leq F_{T_{i}}(t) \leq F_{T_{1}}(t)+F_{T_{2}}(t)-F_{T_{1}, T_{2}}(t, t) .
$$

In that case, $F_{T_{i}}(t)$ can be represented as a convex combination of failure probabilities series and parallel systems. Then, we have

$$
\begin{aligned}
& \lambda\left(F_{T_{1}}(t)+F_{T_{2}}(t)-F_{T_{1}, T_{2}}(t, t)\right)+(1-\lambda) F_{T_{1}, T_{2}}(t, t) \\
= & \lambda\left(F_{T_{1}}(t)+F_{T_{2}}(t)\right)+(1-2 \lambda) F_{T_{1}, T_{2}}(t, t),
\end{aligned}
$$

where the combination parameter $\lambda \in[0,1]$. This latter well-defined statement can derive numerous univariate distribution functions with respect to combination parameter $\lambda$.

In the latter equation, if the distributions of random variables $T_{1}$ and $T_{2}$ are assumed to be identical, namely, $F_{T_{1}}(t)=F_{T_{2}}(t)$, then the new distribution with the parameter set $\Theta$ is given by

$$
G(t ; \Theta)=2 \lambda F_{T_{i}}(t)+(1-2 \lambda) F_{T_{1}, T_{2}}(t, t) .
$$

If transformation $\lambda=\frac{\delta+1}{2}$ is done, range will change from $[0,1]$ to $[-1,1]$. So, for $|\delta| \leq 1$, the distribution function can be written as

$$
\begin{aligned}
G(t ; \Theta) & =(1+\delta) F_{T_{i}}(t)-\delta F_{T_{1}, T_{2}}(t, t) \\
& =(1+\delta) F_{T_{i}}(t)-\delta\left(2 F_{T_{i}}(t)+S_{T_{1}, T_{2}}(t, t)-1\right) \\
& =(1-\delta)\left(1-S_{T_{i}}(t)\right)+\delta\left(1-S_{T_{1}, T_{2}}(t, t)\right) .
\end{aligned}
$$

So, if the distributions of random variables $T_{1}$ and $T_{2}$ are taken independent, namely, $F_{T_{1}, T_{2}}(t, t)=\left(F_{T_{i}}(t)\right)^{2}$ in the first equation of 2 , we can obtain the transmuted distribution constructed by the quadratic rank transmutation method of 27. which has become very popular in the recent years. 
In particular, for $\delta=0$ it gives the baseline distribution $F_{T_{i}}(t)$, for $\delta=-1$, it gives the distribution of the maximum of dependent two random variables with joint distribution function $F_{T_{1}, T_{2}}(t, t)$ and for $\delta=1,2 F_{T_{i}}(t)-F_{T_{1}, T_{2}}(t, t)$ is the distribution of the minimum of two random variables $T_{1}$ and $T_{2}$ with identically distributed.

Theorem 1. The probability density function (p.d.f.) of $T$ is represented in terms of the conditional hazard rates of the component lifetimes $T_{1}$ and $T_{2}$ as

$$
g(t ; \Theta)=(1-\delta) f_{T_{i}}(t)+\delta S_{T_{1}, T_{2}}(t, t)\left(\psi_{1}(t)+\psi_{2}(t)\right),
$$

where $\psi_{1}(t)$ and $\psi_{2}(t)$ denote the failure rates of the corresponding components, given that both components are alive at time $t$.

Proof. The p.d.f. of this distribution can be obtained with derivation of distribution function defined in 2 as follows

$$
g(t ; \Theta)=\frac{d}{d t} G(t ; \Theta)=(1-\delta)\left(\frac{-d}{d t} S_{T_{i}}(t)\right)+\delta\left(\frac{-d}{d t} S_{T_{1}, T_{2}}(t, t)\right)
$$

and the result in $(3)$ will be obtained from the following method.

$$
\begin{aligned}
\frac{-d}{d t} S_{T_{1}, T_{2}}(t, t) & =\frac{-d}{d t} \int_{t}^{\infty} \int_{t}^{\infty} f_{T_{1}, T_{2}}(u, v) d v d u \\
& =-\int_{t}^{\infty} f_{T_{1}, T_{2}}(u, t) d u-\int_{t}^{\infty} f_{T_{1}, T_{2}}(t, v) d v \\
& =-f_{T_{2}}(t) \operatorname{Pr}\left(T_{1} \leq t T_{2}=t\right)-f_{T_{1}}(t) \operatorname{Pr}\left(T_{2} \leq t T_{1}=t\right) \\
& =\left.\frac{-d}{d t_{2}} S_{T_{1}, T_{2}}\left(t, t_{2}\right)\right|_{t_{2}=t}+\left.\frac{-d}{d t_{1}} S_{T_{1}, T_{2}}\left(t_{1}, t\right)\right|_{t_{1}=t} \\
& =\psi_{2}(t) S_{T_{1}, T_{2}}(t, t)+\psi_{1}(t) S_{T_{1}, T_{2}}(t, t)
\end{aligned}
$$

where $\psi_{1}(t)$ and $\psi_{2}(t)$ denote the failure rates of the corresponding components, given that both components are alive at time $t$ and defined as follows:

$\psi_{1}(t)=\lim _{\Delta t \rightarrow 0^{+}} \frac{\operatorname{Pr}\left(t<T_{1} \leq t+\Delta t \mid T_{1}>t, T_{2}>t\right)}{\Delta t}=\frac{\left.\frac{-d}{d t_{1}} S_{T_{1}, T_{2}}\left(t_{1}, t\right)\right|_{t_{1}=t}}{S(t, t)}, \quad t \geq 0$
$\psi_{2}(t)=\lim _{\Delta t \rightarrow 0^{+}} \frac{\operatorname{Pr}\left(t<T_{2} \leq t+\Delta t \mid T_{1}>t, T_{2}>t\right)}{\Delta t}=\frac{\left.\frac{-d}{d t_{2}} S_{T_{1}, T_{2}}\left(t, t_{2}\right)\right|_{t_{2}=t}}{S(t, t)} . \quad t \geq 0$

(See [26] and see [17]).

2.1. Survival and Hazard Rate Functions of Proposed Distribution. The survival function denoted by $S(t ; \Theta)$ of this distribution is defined as follows,

$$
\begin{aligned}
S(t ; \Theta) & =1-G(t ; \Theta)=1-(1+\delta) F_{T_{i}}(t)+\delta F_{T_{1}, T_{2}}(t, t) \\
& =1-(1-\delta)\left(1-S_{T_{i}}(t)\right)-\delta\left(1-S_{T_{1}, T_{2}}(t, t)\right)
\end{aligned}
$$




$$
=(1-\delta) S_{T_{i}}(t)+\delta S_{T_{1}, T_{2}}(t, t) .
$$

The hazard rate function (hrf) corresponding to $(2)$ and $(3)$ is given by

$$
\begin{aligned}
h(t ; \Theta) & =\frac{g(t ; \Theta)}{S(t ; \Theta)}=\frac{(1-\delta) h_{T_{1}}(t) S_{T_{i}}(t)+\delta S_{T_{1}, T_{2}}(t, t)\left(\psi_{1}(t)+\psi_{2}(t)\right)}{(1-\delta) S_{T_{i}}(t)+\delta S_{T_{1}, T_{2}}(t, t)} \\
& =\psi_{1}(t)+\psi_{2}(t)+\frac{(1-\delta) S_{T_{i}}(t)\left(h_{T_{1}}(t)-\left(\psi_{1}(t)+\psi_{2}(t)\right)\right)}{(1-\delta) S_{T_{i}}(t)+\delta S_{T_{1}, T_{2}}(t, t)} \\
& =h_{T_{1}}(t)+\frac{\delta S_{T_{1}, T_{2}}(t, t)\left(\left(\psi_{1}(t)+\psi_{2}(t)\right)-h_{T_{1}}(t)\right)}{(1-\delta) S_{T_{i}}(t)+\delta S_{T_{1}, T_{2}}(t, t)} \\
& =w_{1}(t) h_{T_{1}}(t)+w_{2}(t)\left(\psi_{1}(t)+\psi_{2}(t)\right),
\end{aligned}
$$

where $w_{1}(t)=\frac{(1-\delta) S_{T_{i}}(t)}{(1-\delta) S_{T_{i}}(t)+\delta S_{T_{1}, T_{2}}(t, t)}$ and $w_{1}(t)+w_{2}(t)=1$. Thus, the hrf can be written as a weighted sum of the hrf of the random variable $T_{1}$ and sum of the conditional failure rates of the corresponding components $\left(\psi_{1}(t)+\psi_{2}(t)\right)$.

In the next section, we will introduce a bivariate version of the exponential distribution named the Gumbel bivariate exponential distribution. On the basis of this, the Gumbel univariate exponential distribution is defined and examined. Then, the transmuted Gumbel univariate exponential distribution is taken as a special case for the proposed distribution and some mathematical properties are studied.

\section{Special Case: Transmuted Gumbel Univariate Exponential (TGUE) DisTRIBUTION}

We will first introduce distributions related to setting-up a special case. Then the baseline distribution is defined and we study on some reliability properties such as survival, cumulative hazard rate, hazard rate and mean residual life functions. Moment generating function and moments of proposed distribution are analyzed. ML estimation of model parameters are performed and asymptotic distribution of the parameters are obtained in terms of observed Fisher Information and then asymptotic confidence intervals are also obtained. General expressions for the Rényi entropy is presented. Furthermore, general results for the order statistics of the TGUE random variables are derived.

\subsection{Gumbel Bivariate and Univariate Exponential Distribution.}

3.1.1. Gumbel Bivariate Exponential Distribution. Exponential distribution plays a central role in life testing, reliability and analyses of survival or lifetime data. The Gumbel bivariate exponential (GBE) distribution introduced by [15] is the most popular model for analyzing lifetime data and its survival function is

$$
S_{T_{1}, T_{2}}\left(t_{1}, t_{2}\right)=e^{-\left(\alpha_{1} t_{1}+\alpha_{2} t_{2}+\beta t_{1} t_{2}\right)}, t_{1}, t_{2}>0,
$$


where $\alpha_{1}$ and $\alpha_{2}$ are the scale parameters representing the characteristic life and also positive, $\beta$ is dependency parameter and $0 \leq \beta \leq \alpha_{1} \alpha_{2}$. The marginal survival functions of $T_{1}$ and $T_{2}$ respectively are $e^{-\alpha_{1} t_{1}}$ and $e^{-\alpha_{2} t_{2}}$. Hence $T_{1}$ and $T_{2}$ have exponential marginals. The p.d.f. of the three-parameter GBE distribution corresponding to 4 is given by

$$
\begin{aligned}
f_{T_{1}, T_{2}}\left(t_{1}, t_{2}\right) & =\frac{\partial^{2}}{\partial t_{1} \partial t_{2}} S_{T_{1}, T_{2}}\left(t_{1}, t_{2}\right)=\frac{\partial}{\partial t_{1}}\left(\frac{\partial}{\partial t_{2}} e^{-\left(\alpha_{1} t_{1}+\alpha_{2} t_{2}+\beta t_{1} t_{2}\right)}\right) \\
& =\left(\alpha_{1}+\beta t_{2}\right)\left(\alpha_{2}+\beta t_{1}\right) e^{-\left(\alpha_{1} t_{1}+\alpha_{2} t_{2}+\beta t_{1} t_{2}\right)}, t_{1}, t_{2}>0 .
\end{aligned}
$$

3.1.2. Gumbel Univariate Exponential Distribution. By letting $\alpha_{1}=\alpha_{2}$ and considering the diagonal section of $S_{T_{1}, T_{2}}\left(t_{1}, t_{2}\right)$ i.e., $t_{1}=t_{2}=t$ in the survival function of GBE distribution defined in (4). Then the random vector $\left(T_{1}, T_{2}\right)$ has the Gumbel univariate exponential (GUE) distribution, and the survival function of the GUE distribution can be written as follows

$$
S_{T_{1}, T_{2}}(t, t)=e^{-\left(2 \alpha t+\beta t^{2}\right)}, \quad t>0, \alpha>0,0 \leq \beta \leq \alpha^{2} .
$$

By using the known relation between $S_{T_{1}, T_{2}}(t, t)$ and $F_{T_{1}, T_{2}}(t, t)$, the distribution function of the GUE random variable is given by

$$
F_{T_{1}, T_{2}}(t, t)=1-2 S_{T_{i}}(t)+S_{T_{1}, T_{2}}(t, t)=1-2 e^{-\alpha t}+e^{-\left(2 \alpha t+\beta t^{2}\right)},
$$

and its p.d.f. of the GUE random variable reduces to

$$
\begin{aligned}
f_{T_{1}, T_{2}}(t, t) & =2 \alpha e^{-\alpha t}-(2 \alpha+2 \beta t) e^{-\left(2 \alpha t+\beta t^{2}\right)} \\
& =2 \alpha\left(e^{-\alpha t}-e^{-\left(2 \alpha t+\beta t^{2}\right)}\right)-2 \beta t e^{-\left(2 \alpha t+\beta t^{2}\right)} .
\end{aligned}
$$

The moment generating function of the GUE random variable is given as follows

$$
M_{T}(k)=\int_{0}^{\infty} e^{k T} f_{T_{1}, T_{2}}(t, t) d t=\frac{\alpha+k}{\alpha-k}-\frac{k}{2} \sqrt{\frac{\pi}{\beta}} e^{\frac{(2 \alpha-k)^{2}}{4 \beta}} \operatorname{erfc}\left(\frac{2 \alpha-k}{\sqrt{\beta}}\right),
$$

where $\operatorname{erfc}$ is a complementary error function and $k<\alpha$.

Especially, the first four moments of the GUE random variable $T$ are given as

$$
\begin{aligned}
E(T) & =2\left(-\left.t e^{-\alpha t}\right|_{0} ^{\infty}+\int_{0}^{\infty} e^{-\alpha t} d t+\left.t e^{-\left(2 \alpha t+\beta t^{2}\right)}\right|_{0} ^{\infty}-\int_{0}^{\infty} e^{-\left(2 \alpha t+\beta t^{2}\right)} d t\right) \\
& =2\left(\left.\frac{-1}{\alpha} e^{-\alpha t}\right|_{0} ^{\infty}-e^{\frac{\alpha^{2}}{\beta}} \int_{0}^{\infty} e^{-\left(\sqrt{\beta}\left(t+\frac{\alpha}{\beta}\right)\right)^{2}} d t\right)=\frac{2}{\alpha}-\xi(\alpha, \beta), \\
E\left(T^{2}\right) & =\frac{4}{\alpha^{2}}-\frac{1}{\beta}+\frac{1}{\beta} \xi(\alpha, \beta), \\
E\left(T^{3}\right) & =\frac{12}{\alpha^{3}}-\frac{3 \alpha}{\beta^{2}}+\frac{3}{4 \beta}\left(1+\frac{2 \alpha^{2}}{\beta}\right) \xi(\alpha, \beta),
\end{aligned}
$$




$$
E\left(T^{4}\right)=\frac{48}{\alpha^{4}}-\frac{1}{2 \beta^{2}}\left(4+12 \alpha-\frac{4 \alpha^{2}}{\beta}\right)+\left(3 \frac{\alpha}{\beta^{2}} e^{\frac{\alpha^{2}}{\beta}}+2\left(\frac{\alpha}{\beta}\right)^{3} \xi(\alpha, \beta)\right)
$$

where $\xi(\alpha, \beta)=\sqrt{\frac{\pi}{\beta}} e^{\frac{\alpha^{2}}{\beta}} \operatorname{erfc}\left(\frac{\alpha}{\sqrt{\beta}}\right)$.

3.1.3. Transmuted Gumbel Univariate Exponential Distribution. The transmuted Gumbel univariate exponential (TGUE) distribution is an extended model to analyze more complex data. $T_{1}$ and $T_{2}$ have a exponential distribution with the same shape parameter $\alpha$ and random vector $\left(T_{1}, T_{2}\right)$ has a Gumbel univariate exponential distribution with $\alpha$ and $\beta$ parameters, then we can write

$$
\left\{\begin{array}{l}
S_{T_{i}}(t)=e^{-\alpha t}, \quad F_{T_{i}}(t)=1-e^{-\alpha t} \\
S_{T_{1}, T_{2}}(t, t)=e^{-\left(2 \alpha t+\beta t^{2}\right)}, \quad F_{T_{1}, T_{2}}(t, t)=1-2 e^{-\alpha t}+e^{-\left(2 \alpha t+\beta t^{2}\right)} .
\end{array}\right.
$$

By using equation (2) and (5), the distribution function of the TGUE random variable with the parameter space $\Theta=\left\{(\alpha, \beta, \delta): \alpha>0, \beta<\alpha^{2},-1 \leq \delta \leq 1\right\}$, can be obtained as

$$
\begin{aligned}
G(t ; \Theta) & =(1-\delta)\left(1-S_{T_{i}}(t)\right)+\delta\left(1-S_{T_{1}, T_{2}}(t, t)\right), \\
& =(1-\delta)\left(1-e^{-\alpha t}\right)+\delta\left(1-e^{-\left(2 \alpha t+\beta t^{2}\right)}\right) \\
& =1-(1-\delta) e^{-\alpha t}-\delta e^{-\left(2 \alpha t+\beta t^{2}\right)} .
\end{aligned}
$$

Henceforth, the p.d.f. corresponding to (3) and (6) becomes

$$
g(t ; \Theta)=\frac{d}{d t} G(t ; \Theta)=(1-\delta) f_{T_{i}}(t)+\delta S_{T_{1}, T_{2}}(t, t)\left(\psi_{1}(t)+\psi_{2}(t)\right),
$$

where $\psi_{1}(t)=\frac{\left.\frac{-d}{d t_{1}} e^{-\left(\alpha t_{1}+\alpha t+\beta t_{1} t\right)}\right|_{t_{1}=t}}{e^{-\left(2 \alpha t+\beta t^{2}\right)}}=\alpha+\beta t, \quad \psi_{2}(t)=\alpha+\beta t$ and $\beta \leq \alpha^{2}$. Consequently, the p.d.f. of the TGUE random variable can be written as follows

$$
\begin{aligned}
g(t ; \Theta) & =(1-\delta) \alpha e^{-\alpha t}+\delta e^{-\left(2 \alpha t+\beta t^{2}\right)}(\alpha+\beta t+\alpha+\beta t), \\
& =(1-\delta) \alpha e^{-\alpha t}+\delta(2 \alpha+2 \beta t) e^{-\left(2 \alpha t+\beta t^{2}\right)} .
\end{aligned}
$$

The shapes of the p.d.f. of the TGUE random variable can be analyzed as follows

$$
g^{\prime}(t ; \Theta)=-(1-\delta) \alpha^{2} e^{-\alpha t}-\delta(2 \alpha+2 \beta t)^{2} e^{-\left(2 \alpha t+\beta t^{2}\right)},
$$

by examining this derivation, it is clear that when $0 \leq \delta \leq 1, g^{\prime}(t ; \Theta)<0$ is obtained and we can say that the p.d.f. is decreasing. Also, in order for p.d.f. to be unimodal, it must be $-1 \leq \delta \leq 0$. 

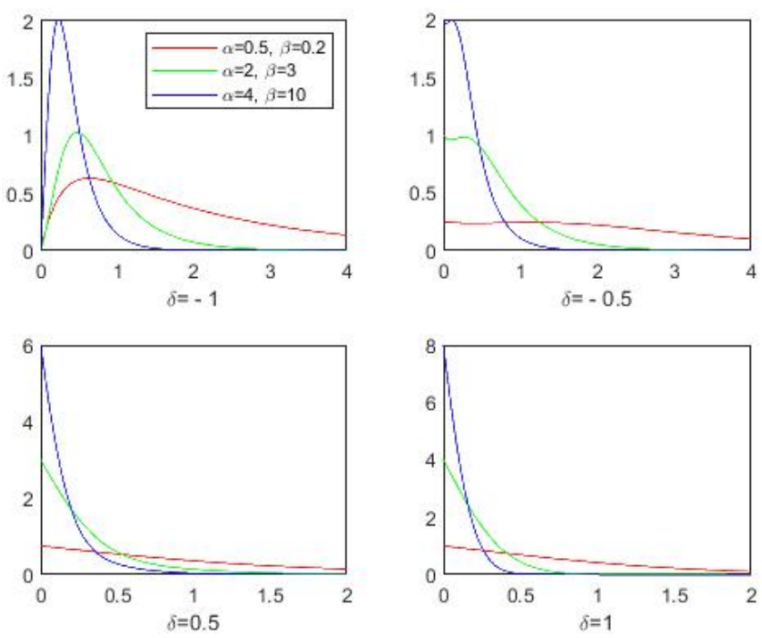

Figure 1. Plots of the TGUE Probability Density Function

3.1.4. Survival, Cumulative Hazard Rate and Hazard Rate Functions of the TGUE Distribution. The survival function of the TGUE random variable is given by

$$
S(t ; \Theta)=1-G(t ; \Theta)=(1-\delta) e^{-\alpha t}+\delta e^{-\left(2 \alpha t+\beta t^{2}\right)} .
$$

Many generalized probability models have been proposed in reliability literature through the fundamental relationship between the cumulative hazard function $H(t ; \Theta)$ and the survival function $S(t ; \Theta)$ is given by

$$
H(t ; \Theta)=-\log S(t ; \Theta)=-\log \left((1-\delta) e^{-\alpha t}+\delta e^{-\left(2 \alpha t+\beta t^{2}\right)}\right) .
$$

Thus, we find the cumulative hazard function of the TGUE random variable and this function describes how the risk of a particular outcome changes with time. We know

$$
H(0 ; \Theta)=0, \lim _{t \rightarrow \infty} H(t ; \Theta)=\infty, H(t ; \Theta)
$$

is increasing for all $t \geq 0$.

The other characteristic of a random variable is the hrf. By using (7) and (8), this function is given as follows

$$
\begin{aligned}
h(t ; \Theta) & =\frac{g(t ; \Theta)}{S(t ; \Theta)}=\frac{(1-\delta) \alpha e^{-\alpha t}+\delta(2 \alpha+2 \beta t) e^{-\left(2 \alpha t+\beta t^{2}\right)}}{(1-\delta) e^{-\alpha t}+\delta e^{-\left(2 \alpha t+\beta t^{2}\right)}} \\
& =\frac{\alpha(1-\delta) e^{\alpha t+\beta t^{2}}+2 \delta(\alpha+\beta t)}{(1-\delta) e^{\alpha t+\beta t^{2}}+\delta} \\
& =(2 \alpha+2 \beta t)-\frac{(1-\delta)(\alpha+2 \beta t) e^{-\alpha t}}{(1-\delta) e^{-\alpha t}+\delta e^{-\left(2 \alpha t+\beta t^{2}\right)}} .
\end{aligned}
$$


The hrf of the TGUE random variable has the following properties:

$$
\begin{gathered}
h(0 ; \Theta)=(1+\delta) \alpha, \\
\delta \neq 1: \lim _{t \rightarrow \infty} h(t ; \Theta)=\lim _{t \rightarrow \infty} \frac{(1-\delta) \alpha e^{-\alpha t}+\delta(2 \alpha+2 \beta t) e^{-\left(2 \alpha t+\beta t^{2}\right)}}{(1-\delta) e^{-\alpha t}+\delta e^{-\left(2 \alpha t+\beta t^{2}\right)}}=\alpha, \\
\delta=1: \lim _{t \rightarrow \infty} h(t ; \Theta)=\lim _{t \rightarrow \infty}(2 \alpha+2 \beta t)=\infty .
\end{gathered}
$$

The hazard rate function will be examined in the extreme values of the parameters:

(1) If $\delta=0$, the hrf is the same as the exponential distribution;

$$
h(t ; \Theta)=\alpha
$$

(2) If $\delta=1$, the hrf is the same as the linear hazard rate function;

$$
h(t ; \Theta)=2(\alpha+\beta t)
$$

(3) If $\beta=0$, the hrf is the same as the transmuted exponential distribution;

$$
h(t ; \Theta)=\frac{(1-\delta) \alpha e^{-\alpha t}+2 \delta \alpha}{(1-\delta) e^{-\alpha t}+\delta} .
$$

Let's investigate the monotonicity of hrf,

$$
h^{\prime}(t ; \Theta)=\frac{-\delta(1-\delta)(\alpha+2 \beta t)^{2} e^{-\left(3 \alpha t+\beta t^{2}\right)}}{\left((1-\delta) e^{-\alpha t}+\delta e^{\left.-\left(2 \alpha t+\beta t^{2}\right)\right)^{2}}\right.} .
$$

It is clear from above derivation, when $-1 \leq \delta \leq 0$, the hazard rate function is increasing, that is, $h^{\prime}(t ; \Theta) \geq 0$. When $0 \leq \delta \leq 1$, the hazard rate function is decreasing $\left(h^{\prime}(t ; \Theta) \leq 0\right)$. Some possible shapes of hrf for selected parameter value are shown in the following figures.

Figure 3.2 shows the hrf defined in $(10)$ with different choices of parameters. This

distribution has an increasing hrf for $-1 \leq \delta \leq 0$. If $0 \leq \delta \leq 1$, the hrf is

$$
\text { decreasing. }
$$

3.1.5. Mean Residual Life Function of the TGUE Random Variable. In this section, we will find the mean residual life $(\mathrm{mrl})$ function of the TGUE random variable which is another important characteristic of a random variable.

$$
\begin{aligned}
m(t ; \Theta) & =E(T-t \mid T>t)=\int_{0}^{\infty}(k-t) d P(T \leq k \mid T>t) \\
& =\frac{\int_{t}^{\infty} S(k ; \Theta) d k}{S(t ; \Theta)}=\frac{(1-\delta) \frac{1}{\alpha} e^{-\alpha t}-\frac{\delta}{2} \sqrt{\frac{\pi}{\beta}} e^{\frac{\alpha^{2}}{\beta}} \operatorname{erfc}\left(\frac{\alpha+\beta t}{\sqrt{\beta}}\right)}{(1-\delta) e^{-\alpha t}+\delta e^{-\left(2 \alpha t+\beta t^{2}\right)}} .
\end{aligned}
$$

The mrl function of the TGUE random variable has the following properties:

(1) If $\delta=0$, the mrl function is the same as the exponential distribution;

$$
m(t ; \Theta)=\frac{1}{\alpha}
$$



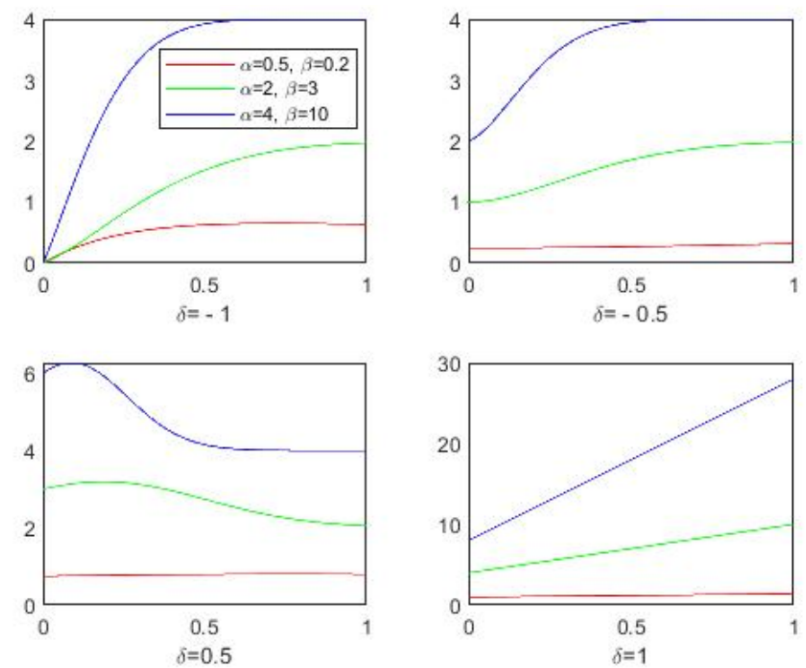

Figure 2. Plots of the TGUE Hazard Rate Function

(2) If $\delta=1$, the mrl function is;

$$
m(t ; \Theta)=-\frac{1}{2} \sqrt{\frac{\pi}{\beta}} \operatorname{erfc}\left(\frac{\alpha+\beta t}{\sqrt{\beta}}\right) e^{\frac{\alpha^{2}}{\beta}+2 \alpha t+\beta t^{2}},
$$

and some possible shapes of 11 for selected parameter values is showed in the following figures.

3.1.6. 3.5. Moment Generating Function and moments of the TGUE Random Variable. In this section, we derive the moment generating function and first four moments for the TGUE distribution. Let $T$ have the TGUE distribution, then the moment generating function of $T$ is given by

$M_{T}(k)=E\left(e^{k T}\right)=(1-\delta) \frac{\alpha}{\alpha-k}+\delta\left(1+\frac{k}{2} \sqrt{\frac{\pi}{\beta}} e^{\frac{(2 \alpha-k)^{2}}{4 \beta}} \operatorname{erfc}\left(\frac{2 \alpha-k}{\sqrt{\beta}}\right)\right), k<\alpha$

The expressions for the expected value and variance are

$$
\begin{gathered}
E(T)=(1-\delta) \frac{1}{\alpha}-\delta\left(e^{\frac{\alpha^{2}}{\beta}} \int_{0}^{\infty} e^{-\left(\sqrt{\beta}\left(t+\frac{\alpha}{\beta}\right)\right)^{2}} d t\right)=(1-\delta) \frac{1}{\alpha}-\delta \xi(\alpha, \beta), \\
E\left(T^{2}\right)=(1-\delta) \frac{2}{\alpha^{2}}+\delta\left(\frac{1}{\beta}-\frac{1}{\beta} \xi(\alpha, \beta)\right) \\
\operatorname{Var}(T)=(1-\delta) \frac{2}{\alpha^{2}}+\delta\left(\frac{1}{\beta}-\frac{1}{\beta} \xi(\alpha, \beta)\right)-\left((1-\delta) \frac{1}{\alpha}-\delta \xi(\alpha, \beta)\right)^{2} .
\end{gathered}
$$



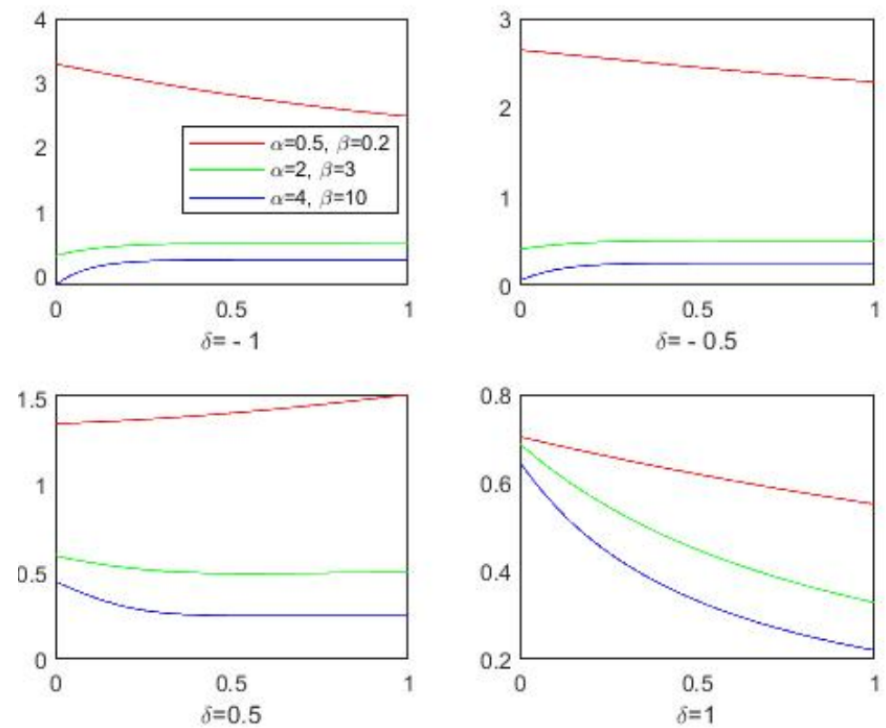

Figure 3. Plots of the TGUE Mean Residual Life Function

Finally, the $3^{\text {th }}$ and $4^{\text {th }}$ moments of the TGUE random variable are obtained as

$$
\begin{gathered}
E\left(T^{3}\right)=(1-\delta) \frac{6}{\alpha^{3}}+\delta\left(\frac{3 \alpha}{\beta^{2}}-\frac{3}{4 \beta}\left(1+\frac{2 \alpha^{2}}{\beta}\right) \xi(\alpha, \beta)\right) \\
E\left(T^{4}\right)=(1-\delta) \frac{24}{\alpha^{4}}+\delta\left(\frac{1}{2 \beta^{2}}\left(4+12 \alpha-\frac{4 \alpha^{2}}{\beta}\right)+\frac{\alpha}{\beta^{2}}\left(3 e^{\frac{\alpha^{2}}{\beta}}+\frac{2 \alpha^{2}}{\beta} \xi(\alpha, \beta)\right)\right) .
\end{gathered}
$$

3.1.7. Estimation by Maximum Likelihood and the Information Matrix of the TGUE Distribution. Let $\left(t_{1}, t_{2}, \cdots, t_{n}\right)$ be sample values from this distribution with parameters $\alpha, \beta$ and $\delta$. The likelihood function for $\Theta=\{\alpha, \beta, \delta\}$ is given by

$$
L\left(\Theta ; t_{1}, t_{2}, \cdots, t_{n}\right)=\prod_{i=1}^{n}\left((1-\delta) \alpha e^{-\alpha t_{i}}+\delta\left(2 \alpha+2 \beta t_{i}\right) e^{-\left(2 \alpha t_{i}+\beta t_{i}^{2}\right)}\right) .
$$

Throughout this subsection, the log-likelihood function is denoted by $l=\log L\left(\Theta ; t_{1}, t_{2}, \cdots, t_{n}\right)$ for brevity. We differentiate $l$ with respect to $\alpha, \beta$ and $\delta$ as follows

$$
\frac{\partial l}{\partial \alpha}=\sum_{i=1}^{n} \frac{-(1-\delta) \alpha^{2} e^{-\alpha t_{i}}+2 \delta\left(1-2 \alpha t_{i}-2 \beta t_{i}^{2}\right) e^{-\left(2 \alpha t_{i}+\beta t_{i}^{2}\right)}}{g\left(t_{i} ; \Theta\right)},
$$




$$
\begin{aligned}
& \frac{\partial l}{\partial \beta}=\sum_{i=1}^{n} \frac{2 \delta t_{i}\left(1-\alpha t_{i}-\beta t_{i}^{2}\right) e^{-\left(2 \alpha t_{i}+\beta t_{i}^{2}\right)}}{g\left(t_{i} ; \Theta\right)}, \\
& \frac{\partial l}{\partial \delta}=\sum_{i=1}^{n} \frac{-\alpha e^{-\alpha t_{i}}+\left(2 \alpha+2 \beta t_{i}\right) e^{-\left(2 \alpha t_{i}+\beta t_{i}^{2}\right)}}{g\left(t_{i} ; \Theta\right)} .
\end{aligned}
$$

The maximum likelihood estimators as $\hat{\alpha}, \hat{\beta}$ and $\hat{\delta}$ are obtained by equating these three equations $(12),(13)$ and $(14)$ to zero and solving the equations simultaneously. For these three parameters, we will get the second order derivatives of logarithms of the likelihood function for obtaining the elements of the Fisher-Information Matrix.

$$
\begin{aligned}
& I_{\alpha \alpha}=\frac{\partial^{2} l}{\partial \alpha^{2}}=-\sum_{i=1}^{n} \frac{(1-\delta)^{2} e^{-2 \alpha t_{i}}-2 \delta(1-\delta)\left(\alpha \beta t_{i}^{3}+\left(\alpha^{2}+2 \beta\right) t_{i}^{2}-2\right) e^{-\left(3 \alpha t_{i}+\beta t_{i}^{2}\right)}+4 \delta^{2} e^{-2\left(2 \alpha t_{i}+\beta t_{i}^{2}\right)}}{\left(g\left(t_{i} ; \Theta\right)\right)^{2}} \\
& I_{\beta \beta}=\frac{\partial^{2} l}{\partial \beta^{2}}=-\sum_{i=1}^{n} \frac{2 \delta(1-\delta) t_{i}^{3} \alpha\left(2-\alpha t_{i}-\beta t_{i}^{2}\right) e^{-\left(3 \alpha t_{i}+\beta t_{i}^{2}\right)}+4 \delta^{2} t_{i}^{2} e^{-2\left(2 \alpha t_{i}+\beta t_{i}^{2}\right)}}{\left(g\left(t_{i} ; \Theta\right)\right)^{2}}, \\
& I_{\delta \delta}=\frac{\partial^{2} l}{\partial \delta^{2}}=-\sum_{i=1}^{n}\left(\frac{\alpha e^{-\alpha t_{i}}+2\left(\alpha+\beta t_{i}\right) e^{-\left(2 \alpha t_{i}+\beta t_{i}^{2}\right)}}{g\left(t_{i} ; \Theta\right)}\right)^{2}, \\
& I_{\alpha \beta}=I_{\beta \alpha}=\frac{\partial^{2} l}{\partial \beta \partial \alpha} \\
& =-\sum_{i=1}^{n}\left(\frac{2 \delta(1-\delta) t_{i}\left(1+\alpha t_{i}-\left(\alpha^{2}+\beta\right) t_{i}^{2}-\alpha \beta t_{i}^{3}\right) e^{-\left(3 \alpha t_{i}+\beta t_{i}^{2}\right)}+4 \delta^{2} t_{i} e^{-2\left(2 \alpha t_{i}+\beta t_{i}^{2}\right)}}{\left(g\left(t_{i} ; \Theta\right)\right)^{2}}\right), \\
& I_{\alpha \delta}=I_{\delta \alpha}=\frac{\partial^{2} l}{\partial \delta \partial \alpha}=-\sum_{i=1}^{n} \frac{2\left((\alpha+\beta)+\alpha t_{i}\right) t_{i} e^{-\left(3 \alpha t_{i}+\beta t_{i}^{2}\right)}}{\left(g\left(t_{i} ; \Theta\right)\right)^{2}}, \\
& I_{\beta \delta}=I_{\delta \beta}=\frac{\partial^{2} l}{\partial \delta \partial \beta}=-\sum_{i=1}^{n} \frac{-2 \alpha t_{i}\left(1-\alpha t_{i}-\beta t_{i}^{2}\right) e^{-\left(3 \alpha t_{i}+\beta t_{i}^{2}\right)}}{\left(g\left(t_{i} ; \Theta\right)\right)^{2}} .
\end{aligned}
$$

Thus, Fisher information matrix, $I_{n}(\Theta)$ of sample size $n$ for $\Theta$ is as follows:

$$
I_{n}(\Theta)=-E\left(\begin{array}{ccc}
I_{\alpha \alpha} & I_{\alpha \beta} & I_{\alpha \delta} \\
I_{\beta \alpha} & I_{\beta \beta} & I_{\beta \delta} \\
I_{\delta \alpha} & I_{\delta \beta} & I_{\delta \delta}
\end{array}\right)
$$

Inverse of the Fisher-information matrix of single observation, i.e., $I_{1}^{-1}(\Theta)$ indicates asymptotic variance-covariance matrix of maximum likelihood estimates of $\Theta$. Hence, the distribution of maximum likelihood estimator for $\Theta$ is asymptotically normal with mean $\Theta$ and variance-covariance matrix $I_{1}^{-1}(\Theta)$. Namely,

$$
\left[\begin{array}{l}
\hat{\alpha} \\
\hat{\beta} \\
\hat{\delta}
\end{array}\right] \sim A N\left(\left[\begin{array}{l}
\alpha \\
\beta \\
\delta
\end{array}\right], \frac{I_{1}^{-1}(\Theta)}{n}\right)
$$


By solving this inverse dispersion matrix these solutions will yield asymptotic variance and covariance of these ML estimators for these parameters.

We can approximate $100(1-\gamma) \%$ confidence intervals for $\alpha, \beta$ and $\delta$ by using (15) are obtained respectively as

$$
\begin{aligned}
& {\left[\hat{\alpha}-z_{1-\frac{\gamma}{2}} \sqrt{\frac{I_{1_{\alpha \alpha}}^{-1}}{n}}, \hat{\alpha}+z_{1-\frac{\gamma}{2}} \sqrt{\frac{I_{1_{\alpha \alpha}}^{-1}}{n}}\right],} \\
& {\left[\hat{\beta}-z_{1-\frac{\gamma}{2}} \sqrt{\frac{I_{1_{\beta \beta}}^{-1}}{n}}, \hat{\beta}+z_{1-\frac{\gamma}{2}} \sqrt{\frac{I_{1_{\beta \beta}}^{-1}}{n}}\right],} \\
& {\left[\hat{\delta}-z_{1-\frac{\gamma}{2}} \sqrt{\frac{I_{1_{\delta \delta}}^{-1}}{n}}, \hat{\delta}+z_{1-\frac{\gamma}{2}} \sqrt{\frac{I_{1_{\delta \delta}}^{-1}}{n}}\right],}
\end{aligned}
$$

where $z_{1-\frac{\gamma}{2}}$ is the upper $100 \gamma$ the quantile of the standard normal distribution, and $I_{1 . .}^{-1}$ denotes respective diagonal elements of $I_{1}^{-1}$.

3.1.8. Random Number Generation from the TGUE Distribution. Remember the distribution function defined in section 2 ,

$$
G(t)=\lambda\left(F_{T_{1}}(t)+F_{T_{2}}(t)-F_{T_{1}, T_{2}}(t, t)\right)+(1-\lambda) F_{T_{1}, T_{2}}(t, t)
$$

where $0 \leq \lambda \leq 1$. Again, emphasize that $G(t)$ represents a two-component mixture distribution, where the distribution functions of the $T_{\min }$ and $T_{\max }$ are the components of this mixture, respectively. To generate a random number from $G(t)$, we apply the reference Gentle [14 pp.125. Accordingly, a random number $V$ is generated from uniform distribution on $(0,1)$ to decide which of the components are chosen. As a result, when $V \leq \lambda$, the random number will be generated from $F_{T_{m i n}}(t)$ by equating as $F_{T_{m i n}}(t)=V$. Otherwise, namely $V>\lambda$, the random number will be generated from the distribution of $T_{\max }$ by equating $F_{T_{\max }}(t)=V$.

First of all, we will consider how to produce component lifetimes. By citing the method given in Gentle 14 pp.109, these component lifetimes will be generated with the help of the conditional distribution function. Namely, $F_{T_{1}, T_{2}}\left(t_{1}, t_{2}\right)$ can be expressed as the product of the cdf of $T_{1}$ and the conditional cdf of $T_{2}$ with given $T_{1}=t_{1}$, i.e. $F_{T_{1}, T_{2}}\left(t_{1}, t_{2}\right)=F_{T_{1}}\left(t_{1}\right) F_{T_{2} \mid T_{1}}\left(t_{2}\right)$.

In the first step, a random number $U_{1}$ is generated from the uniform distribution on the interval $(0,1)$. Then we generate the lifetime of the first component $t_{1}=F_{T_{1}}^{-1}\left(U_{1}\right)$. In the second step, again we generate a uniformly distributed random variable $U_{2}$ (independent of $\left.U_{1}\right)$ on $(0,1)$. Therefore, the lifetime of the second component can be generated by equating $t_{2}=F_{T_{2} \mid T_{1}=t_{1}}^{-1}\left(U_{2}\right)$. Hence, the random number from the TGUE is generated as for $V \leq \lambda, t=$ 
$\min \hat{a}\left\{t_{1}, t_{2}\right\}$ and for $V>\lambda, t=\max \hat{a}\left\{t_{1}, t_{2}\right\}$. Then, according to the abovementioned steps, $t_{1}=\frac{-1}{\alpha} \ln \left(1-U_{1}\right)$ and $t_{2}=-\frac{\alpha}{\beta}-\frac{W_{-1}(\eta)}{\alpha+\beta t_{1}}$ are generated where $-\left(1-U_{2}\right)\left(\frac{\alpha^{2}}{\beta}+\alpha t_{1}\right) e^{-\left(\frac{\alpha^{2}}{\beta}+\alpha t_{1}\right)}=\eta$. Here $W_{-1}($.$) denotes the lower part of$ Lambert $\mathrm{W}$-function whose domain is $\left[-e^{-1}, 0\right)$ and range $(-\infty,-1]$. A more detailed inference about generating second component lifetime is given in the appendix.

3.1.9. Rényi Entropy of the TGUE Distribution. The entropy of a random variable is a measure of variation of the uncertainty, see 25]. Then the Rényi entropy function of the random variable $T$ with p.d.f. (7) is defined by

$$
I_{R}(\rho)=\frac{1}{1-\rho} \log \int_{0}^{\infty}(g(t ; \Theta))^{\rho} d t
$$

where $\rho>0, \rho \neq 1$. We have the following series representation of $(g(t ; \Theta))^{\rho}$ by applying the generalized Binomial theorem to obtain Rényi entropy for proposed distribution. Accordingly,

$$
(g(t ; \Theta))^{\rho}=\left((1-\delta) \alpha e^{-\alpha t}+\delta(2 \alpha+2 \beta t) e^{-\left(2 \alpha t+\beta t^{2}\right)}\right)^{\rho} .
$$

$(g(t ; \Theta))^{\rho}$ can be written as an infinite series representation as follows.

$$
\begin{aligned}
(g(t ; \Theta))^{\rho} & =\sum_{j=0}^{\infty}\left(\begin{array}{l}
\rho \\
j
\end{array}\right)\left((1-\delta) \alpha e^{-\alpha t}\right)^{\rho-j}\left(\delta(2 \alpha+2 \beta t) e^{-\left(2 \alpha t+\beta t^{2}\right)}\right)^{j} \\
& =\sum_{j=0}^{\infty}\left(\begin{array}{l}
\rho \\
j
\end{array}\right)(1-\delta)^{\rho-j} \alpha^{\rho-j} e^{-(\rho-j) \alpha t} \delta^{j}(2 \alpha+2 \beta t)^{j} e^{-j\left(2 \alpha t+\beta t^{2}\right)} \\
& =\sum_{j=0}^{\infty}\left(\begin{array}{l}
\rho \\
j
\end{array}\right)(1-\delta)^{\rho-j} \delta^{j} \alpha^{\rho-j}(2 \alpha+2 \beta t)^{j} e^{-(\rho+j) \alpha t-j \beta t^{2}}
\end{aligned}
$$

In the latter equation, the statement $e^{-(\rho+j) \alpha t-j \beta t^{2}}$ is rearranged as; $e^{-j \beta\left(t+\frac{(\rho+j) \alpha}{2 j \beta}\right)^{2}+\frac{(\rho+j)^{2} \alpha^{2}}{4 j \beta}}$ and if the Binomial theorem is applied in $(2 \alpha+2 \beta t)^{j}$, we can write

$$
\begin{aligned}
(g(t ; \Theta))^{\rho} & =\sum_{j=0}^{\infty} \sum_{l=0}^{j}\left(\begin{array}{l}
\rho \\
j
\end{array}\right)\left(\begin{array}{l}
j \\
l
\end{array}\right)(1-\delta)^{\rho-j} \delta^{j} 2^{j} \alpha^{\rho-j} \alpha^{j-l} \beta^{l} t^{l} e^{-j \beta\left(t+\frac{(\rho+j) \alpha}{2 j \beta}\right)^{2}+\frac{(\rho+j)^{2} \alpha^{2}}{4 j \beta}} \\
& =\sum_{j=0}^{\infty} \sum_{l=0}^{j}\left(\begin{array}{l}
\rho \\
j
\end{array}\right)\left(\begin{array}{l}
j \\
l
\end{array}\right)(1-\delta)^{\rho-j} \delta^{j} 2^{j} \alpha^{\rho-l} \beta^{l} e^{\frac{(\rho+j)^{2} \alpha^{2}}{4 j \beta}} t^{l} e^{-j \beta\left(t+\frac{(\rho+j) \alpha}{2 j \beta}\right)^{2}}
\end{aligned}
$$

Then, the Rényi entropy can be written as follows

$$
I_{R}(\rho)=\frac{1}{1-\rho} \log \left[\sum_{j=0}^{\infty} \sum_{l=0}^{j}\left(\begin{array}{c}
\rho \\
j
\end{array}\right)\left(\begin{array}{c}
j \\
l
\end{array}\right)(1-\delta)^{\rho-j} \delta^{j} 2^{j} \alpha^{\rho-l} \beta^{l} e^{\frac{(\rho+j)^{2} \alpha^{2}}{4 j \beta}} \int_{0}^{\infty} t^{l} e^{-j \beta\left(t+\frac{(\rho+j) \alpha}{2 j \beta}\right)^{2}} d t\right]
$$


if the transformation $z=j \beta\left(t+\frac{(\rho+j) \alpha}{2 j \beta}\right)^{2}$ is done in above integral,

$$
\int_{0}^{\infty} t^{l} e^{-j \beta\left(t+\frac{(\rho+j) \alpha}{2 j \beta}\right)^{2}} d t=\int_{\frac{(\rho+j)^{2} \alpha^{2}}{4 j \beta}}^{\infty} \frac{1}{2 \sqrt{j \beta z}}\left(\sqrt{\frac{z}{j \beta}}-\frac{(\rho+j) \alpha}{2 j \beta}\right)^{l} e^{-z} d z
$$

and the Binomial expansion is applied for $\left(\sqrt{\frac{z}{j \beta}}-\frac{(\rho+j) \alpha}{2 j \beta}\right)^{l}$ again, then the equality

$$
\left(\sqrt{\frac{z}{j \beta}}-\frac{(\rho+j) \alpha}{2 j \beta}\right)^{l}=\sum_{k=0}^{l}\left(\begin{array}{c}
l \\
k
\end{array}\right)\left(\sqrt{\frac{z}{j \beta}}\right)^{k}\left(\frac{-(\rho+j) \alpha}{2 j \beta}\right)^{l-k}
$$

is obtained, then

$$
\begin{aligned}
\int_{\frac{(\rho+j)^{2} \alpha^{2}}{4 j \beta}}^{\infty} \frac{1}{2 \sqrt{j \beta z}}( & \left.\sqrt{\frac{z}{j \beta}}-\frac{(\rho+j) \alpha}{2 j \beta}\right)^{l} e^{-z} d z \\
& =\frac{1}{2} \sum_{k=0}^{l}\left(\begin{array}{c}
l \\
k
\end{array}\right)\left(\frac{-(\rho+j) \alpha}{2 j \beta}\right)^{l-k} j \beta^{-\frac{k+1}{2}} \int_{\frac{(\rho+j)^{2} \alpha^{2}}{4 j \beta}}^{\infty} z^{\frac{k-1}{2}} e^{-z} d z
\end{aligned}
$$

Thus, the last integral can be expressed in terms of incomplete Gamma function as follows,

$$
\int_{\frac{(\rho+j)^{2} \alpha^{2}}{4 j \beta}}^{\infty} z^{\frac{k-1}{2}} e^{-z} d z=\Gamma\left(\frac{k+1}{2}, \frac{(\rho+j)^{2} \alpha^{2}}{4 j \beta}\right)
$$

Now, we obtain an explicit equality for $I_{R}(\rho)$ as follows,

$$
\begin{gathered}
I_{R}(\rho)=\frac{1}{1-\rho} \log \sum_{j=0}^{\infty} \sum_{l=0}^{j} \sum_{k=0}^{l}\left(\begin{array}{c}
\rho \\
j
\end{array}\right)\left(\begin{array}{l}
j \\
l
\end{array}\right)\left(\begin{array}{l}
l \\
k
\end{array}\right)(-1)^{l-k}(1-\delta)^{\rho-j} \delta^{j} 2^{j+k-l-1} \\
\alpha^{\rho-k} \beta^{\frac{k-1}{2}}(\rho+j)^{l-k} j^{\frac{k-1}{2}-l} e^{\frac{(\rho+j)^{2} \alpha^{2}}{4 j \beta}} \Gamma\left(\frac{k+1}{2}, \frac{(\rho+j)^{2} \alpha^{2}}{4 j \beta}\right)
\end{gathered}
$$

3.1.10. Order Statistics of the TGUE Distribution. The order statistics are among the most basic tools in non-parametric statistics and inference. Also, the order statistics arise in the analysis of reliability of a system and it can represent the lifetimes of components of a reliability system. Let $T_{(1)}, T_{(2)}, \ldots, T_{(n)}$ denote the order statistics of a random sample $T_{1}, T_{2}, \ldots, T_{n}$ from a continuous population with p.d.f. $g(t ; \Theta)$ and distribution function $G(t ; \Theta)$, then the p.d.f. of $j^{t h}$ order statistics $T_{(j)}$ for $j=1,2, \ldots, n$ is given by

$$
\begin{aligned}
f_{T_{(j)}}(t ; \Theta)= & \frac{n !}{(j-1) !(n-j) !} g(t ; \Theta)[G(t ; \Theta)]^{j-1}[1-G(t ; \Theta)]^{n-j} \\
& \frac{n !}{(j-1) !(n-j) !}\left((1-\delta) \alpha e^{-\alpha t}+\delta(2 \alpha+2 \beta t) e^{-\left(2 \alpha t+\beta t^{2}\right)}\right)
\end{aligned}
$$




$$
\times\left(1-(1-\delta) e^{-\alpha t}-\delta e^{-\left(2 \alpha t+\beta t^{2}\right)}\right)^{j-1}\left((1-\delta) e^{-\alpha t}+\delta e^{-\left(2 \alpha t+\beta t^{2}\right)}\right)^{n-j},
$$

therefore, the p.d.f. of the first order statistics $T_{(1)}$ is given by

$$
\begin{aligned}
f_{T_{(1)}}(t ; \Theta)= & n\left((1-\delta) \alpha e^{-\alpha t}+\delta(2 \alpha+2 \beta t) e^{-\left(2 \alpha t+\beta t^{2}\right)}\right) \\
& \times\left[(1-\delta) e^{-\alpha t}+\delta e^{-\left(2 \alpha t+\beta t^{2}\right)}\right]^{n-1},
\end{aligned}
$$

and the p.d.f. of the last order statistics $T_{(n)}$ is given

$$
\begin{aligned}
f_{T_{(n)}}(t ; \Theta)= & n\left((1-\delta) \alpha e^{-\alpha t}+\delta(2 \alpha+2 \beta t) e^{-\left(2 \alpha t+\beta t^{2}\right)}\right) \\
& \times\left(1-(1-\delta) e^{-\alpha t}-\delta e^{-\left(2 \alpha t+\beta t^{2}\right)}\right)^{j-1} .
\end{aligned}
$$

Note that $\delta=0$ yields the order statistics of the exponential distribution with parameter $\alpha$ and when $\delta=1$ yields the order statistics of the TGUE distribution with parameter $(\alpha, \beta)$.

\section{Numerical Examples}

In this section, we provide three data analyses in order to assess the goodness-offit of the TGUE distribution. The following tables show goodness-of-fit measures for the different distributions.

Data Set 1. (Wheaton River Flood Data) The data consist of the exceedances of flood peaks (in $\mathrm{m}^{3} / \mathrm{s}$ ) of the Wheaton River near Carcross in Yukon Territory, Canada. The data consist of 72 exceedances for the years 1958-1984, rounded to one decimal place: $1.7,2.2,14.4,1.1,0.4,20.6,5.3,0.7,13.0,12.0,9.3,1.4,18.7$, $8.5,25.5,11.6,14.1,22.1,1.1,2.5,14.4,1.7,37.6,0.6,2.2,39.0,0.3,15.0,11.0$, $7.3,22.9,1.7,0.1,1.1,0.6,9.0,1.7,7.0,20.1,0.4,14.1,9.9,10.4,10.7,30.0,3.6$, $5.6,30.8,13.3,4.2,25.5,3.4,11.9,21.5,27.6,36.4,2.7,64.0,1.5,2.5,27.4,1.0$, $27.1,20.2,16.8,5.3,9.7,27.5,2.5,27.0,1.9,2.8$. Firstly, these data were analyzed by [10]. Later on, Beta-Pareto (BP) distribution was applied to these data by [2]. Merovcia and Pukab 22] made a comparison between Pareto $(\mathrm{P})$ and Transmuted Pareto (TP) distribution. They showed that better model is the transmuted Pareto distribution. Bourguignon et al. 9] proposed Kumaraswamy Pareto (Kw-P) distribution. Tahir 30] have proposed Weibull-Pareto (WP) distribution and made a comparison with Beta Exponentiated Pareto (BEP) distribution. Nasiru and Luguterah 24] have proposed a different type of Weibull-Pareto (NWP) distribution. Exponential Modified Discrete Lindley (EMDL) distribution was applied to these data by 31 . We fit data to TGUE distribution and get parameter estimates as $\hat{\alpha}=0.0672, \hat{\beta}=0.2972, \hat{\delta}=0.1976 \dddot{\imath}$. According to the model selection criteria (AIC) tabulated in Table 5.1, TGUE takes the first place amongst 9 proposed models. 
Table 5.1. K-S test values, -2LL, AIC and BIC for TGUE, P, TP, EP, BP, Kw-P, WP, BEP, BGP and EMDL distributions

\begin{tabular}{|c|c|c|c|c|}
\hline Model & K-S & -2LL & AIC & BIC \\
\hline TGUE & 0.089 & 496.3 & 502.3 & 509.1 \\
\hline EMDL & 0.116 & 503.6 & 507.6 & 512.1 \\
\hline P & 0.456 & 606.1 & 610.1 & 610.4 \\
\hline TP & 0.389 & 572.4 & 578.4 & 580.9 \\
\hline EP & 0.199 & 574.6 & 578.6 & 583.2 \\
\hline BP & 0.175 & 567.4 & 573.4 & 580.3 \\
\hline Kw-P & 0.170 & 542.4 & 548.4 & 555.3 \\
\hline WP & - & 498.8 & 502.8 & 507.3 \\
\hline BEP & - & 496.1 & 504.1 & 513.2 \\
\hline
\end{tabular}

Data set 2. (Bladder Cancer Application) The second data set on the remission times (in months) of a random sample of 128 bladder cancer patients Lee and Wang 18 is given by $0.08,2.09,3.48,4.87,6.94,8.66,13.11,23.63,0.20,2.23$, $3.52,4.98,6.97,9.02,13.29,0.40,2.26,3.57,5.06,7.09,9.22,13.80,25.74,0.50$, $2.46,3.64,5.09,7.26,9.47,14.24,25.82,0.51,2.54,3.70,5.17,7.28,9.74,14.76$, $26.31,0.81,2.62,3.82,5.32,7.32,10.06,14.77,32.15,2.64,3.88,5.32,7.39,10.34$, $14.83,34.26,0.90,2.69,4.18,5.34,7.59,10.66,15.96,36.66,1.05,2.69,4.23,5.41$, $7.62,10.75,16.62,43.01,1.19,2.75,4.26,5.41,7.63,17.12,46.12,1.26,2.83,4.33$, $5.49,7.66,11.25,17.14,79.05,1.35,2.87,5.62,7.87,11.64,17.36,1.40,3.02,4.34$, $5.71,7.93,11.79,18.10,1.46,4.40,5.85,8.26,11.98,19.13,1.76,3.25,4.50,6.25$, $8.37,12.02,2.02,3.31,4.51,6.54,8.53,12.03,20.28,2.02,3.36,6.76,12.07,21.73$, $2.07,3.36,6.93,8.65,12.63,22.69$. In this section, we test the performance of the TGUE distribution and show it to be an improved model as compared to some of its sub-models such as transmuted inverse Rayleigh distribution (TIRD), transmuted inverted exponential distribution (TIED), inverse Weibull distribution (IWD) and transmuted inverse Weibull distribution (IWD). It is clear from Table 5.2 that the TGUE model provides better fits than other models to this data sets. For the TGUE distribution parameter estimates are $\hat{\alpha}=0.0485, \hat{\beta}=0.0057, \hat{\delta}=0.7745 i^{\sim}$ and this distribution has the lower AIC, BIC and $\mathrm{K}-\mathrm{S}$ values.

Table 5.2. K-S test values, -2LL, AIC and BIC for TGUE, TIW, TIE, IW and TIR distributions

\begin{tabular}{|c|c|c|c|c|}
\hline Model & K-S & -2LL & AIC & BIC \\
\hline TGUE & 0.065 & 824.2 & 830.1 & 838.6 \\
\hline TIW & 0.119 & 877.0 & 879.4 & 879.7 \\
\hline TIE & 0.155 & 885.6 & 889.6 & 889.8 \\
\hline IW & 0.131 & 888.0 & 892.0 & 892.2 \\
\hline TIR & 0.676 & 1420.4 & 1424.4 & 1424.6 \\
\hline
\end{tabular}


Data set 3. (Bank B Data) The data set represents the waiting times (in minutes) before customer service of 60 bank customers in Bank B. This data set is given as: $0.1,0.2,0.3,0.7,0.9,1.1,1.2,1.8,1.9,2.0,2.2,2.3,2.3,2.3,2.5,2.6,2.7,2.7$, $2.9,3.1,3.1,3.2,3.4,3.4,3.5,3.9,4.0,4.2,4.5,4.7,5.3,5.6,5.6,6.2,6.3,6.6$, $6.8,7.3,7.5,7.7,7.7,8.0,8.0,8.5,8.5,8.7,9.5,10.7,10.9,11.0,12.1,12.3,12.8$, $12.9,13.2,13.7,14.5,16.0,16.5,28.0$. This data was analyzed by 3 and was also used by 29. They fit this data to Lindley (L) and generalized Lindley (GL) distributions. We fit data to TGUE distribution and get parameter estimates as $\hat{\alpha}=0.185, \hat{\beta}=0.472, \hat{\delta}=-0.222$. According to the model selection criteria tabulated in Table 5.3, it is said that TUGE takes first place in amongst 3 proposed models.

Table 5.3. K-S test values, -2LL AIC and BIC for TGUE, L and Exp distributions

\begin{tabular}{|c|c|c|c|c|}
\hline Model & K-S & -2LL & AIC & BIC \\
\hline TGUE & 0.067 & 336.777 & 342.777 & 349.060 \\
\hline L & 0.080 & 338.203 & 340.203 & 341.759 \\
\hline GL & 0.068 & 338.026 & 342.026 & 341.582 \\
\hline
\end{tabular}

In the above three tables, it is clear that the values of the Akaike information criterion (AIC) and Bayesian information criterion (BIC) are smaller for the TGUE distribution compared to those values of the other models; the new distribution is a very competitive model to these data.

\section{Conclusion}

In this article, we propose a new model of transmuted distribution so-called the transmuted Gumbel univariate exponential distribution. The subject distribution is generated by using the convex combination of failure probabilities of two-component series and systems and taking the Gumbel univariate exponential distribution as the base distribution. Some mathematical and statistical properties including explicit expressions for the probability density, survival, cumulative hazard rate, hazard rate and mean residual life functions, also, moment generating function and moments are addressed. The estimation of parameters is approached by the maximum likelihood method. According to K-S values in Numerical Examples Section, the applications of the transmuted Gumbel univariate exponential distribution to real data show that the new distribution can be used to provide better fits than the other distributions. We hope that this new distribution may attract wider applications in the lifetime literature. Taking bivariate distributions will guide to derivation of many new univariate distributions. 


\section{APPENDIX}

Conditional cdf of $T_{2}$ with given $T_{1}=t_{1}$ is given by

$$
\begin{aligned}
F_{T_{2} \mid T_{1}}\left(t_{2}\right) & =\frac{\frac{\partial}{\partial t_{1}} F_{T_{1}, T_{2}}\left(t_{1}, t_{2}\right)}{f_{T_{1}}\left(t_{1}\right)}=\frac{\frac{\partial}{\partial t_{1}}\left(1-e^{-\alpha t_{1}}-e^{-\alpha t_{2}}+e^{-\alpha\left(t_{1}+t_{2}\right)-\beta t_{1} t_{2}}\right)}{\alpha e^{-\alpha t_{1}}} \\
& =\frac{\alpha e^{-\alpha t_{1}}-\left(\alpha+\beta t_{2}\right) e^{-\alpha\left(t_{1}+t_{2}\right)-\beta t_{1} t_{2}}}{\alpha e^{-\alpha t_{1}}} \\
& =1-\left(1+\frac{\beta}{\alpha} t_{2}\right) e^{-\left(\alpha+\beta t_{1}\right) t_{2}} .
\end{aligned}
$$

Hence, by equating $F_{T_{2} \mid T_{1}}\left(t_{2}\right)=U_{2}$ where $U_{2}$ is uniformly distributed random variable on the interval $(0,1)$ we have a non linear equation to get solution for $t_{2}$ as follows,

$$
1-\left(1+\frac{\beta}{\alpha} t_{2}\right) e^{-\left(\alpha+\beta t_{1}\right) t_{2}}=U_{2}
$$

To solve the above equation for $t_{2}$, we use Lambert $\mathrm{W}$ - function which is defined as the solution of the equation $W(z) e^{W(z)}=z$, where $z$ is the complex number. If $z$ is any real number, then this equation has a solution on $\left[-e^{-1},+\infty\right)$. In equation $\sqrt{17}$, if the expression $1+\frac{\beta}{\alpha} t_{2}$ is taken as $z$, we can write

$$
z e^{-\left(\frac{\alpha^{2}}{\beta}+\alpha t_{1}\right) z} e^{\left(\frac{\alpha^{2}}{\beta}+\alpha t_{1}\right)}=1-U_{2} .
$$

Multiplying both sides of equation above by $-\left(\frac{\alpha^{2}}{\beta}+\alpha t_{1}\right)$, above expression can be simplified as follows,

$$
-\left(\frac{\alpha^{2}}{\beta}+\alpha t_{1}\right) z e^{-\left(\frac{\alpha^{2}}{\beta}+\alpha t_{1}\right) z}=-\left(1-U_{2}\right)\left(\frac{\alpha^{2}}{\beta}+\alpha t_{1}\right) e^{-\left(\frac{\alpha^{2}}{\beta}+\alpha t_{1}\right)} .
$$

Substituting $-\left(\frac{\alpha^{2}}{\beta}+\alpha t_{1}\right) z=W(z)$, we have the Lambert equation

$$
W(z) e^{-W(z)}=\eta
$$

where $\eta=-\left(1-U_{2}\right)\left(\frac{\alpha^{2}}{\beta}+\alpha t_{1}\right) e^{-\left(\frac{\alpha^{2}}{\beta}+\alpha t_{1}\right)}$. Hence, the solution for $W(z)$ is

$$
-\left(\frac{\alpha^{2}+\alpha \beta t_{1}}{\beta}\right) z=W_{-1}(\eta)
$$

So, $t_{2}$ is found as follows

$$
t_{2}=-\frac{\alpha}{\beta}-\frac{1}{\alpha+\beta t_{1}} W_{-1}(\eta) .
$$

To show the uniqueness of the solution for $t_{2}$ we take into account the well known inequality $e^{-(z+1)} \geq-z$ and replacing $z$ with $-\left(\alpha+\beta t_{1}\right) \frac{\alpha}{\beta}$, then $\eta \geq-\frac{1}{e}$. 
This result guarantees that $\eta$ belongs to domain of negative branch of Lambert W-function.

\section{REFERENCES}

[1] Abd El Hady, N. E., Exponentiated Transmuted Weibull Distribution, International Journal of Mathematical, Computational, Statistical, Natural and Physical Engineering, 8(6) (2014).

[2] Akinsete, A., Famoye, F. and Lee, C., The Beta-Pareto distribution, Statistics, 42(6) (2008) 547-563.

[3] Al-Mutairi, D. K., Ghitany, M. E., and Kundu, D., Inferences on stress-strength reliability from Lindley distributions, Communications in Statistics-Theory and Methods, 42 (8), (2013), 1443-1463.

[4] Aryal, G. R. and Tsokos, C. P., On the transmuted extreme value distribution with application, Nonlinear Analysis: Theory, Methods \& Applications, 71(12) (2009), 1401-1407.

[5] Aryal, G. R. and Tsokos, C. P., Transmuted Weibull Distribution: A Generalization of the Weibull Probability Distribution, European Journal of Pure and Applied Mathematics, 4(2) (2011), 89-102.

[6] Aryal, G. R., Transmuted log-logistic distribution, Journal of Statistics Applications 6 Probability, 2(1) (2013), 11-20.

[7] Ashour, S. K. and Eltehiwy, M. A., Transmuted Lomax distribution, American Journal of Applied Mathematics and Statistics, 1(6) (2013a), 121-127.

[8] Ashour, S. K. and Eltehiwy, M. A., Transmuted exponentiated Lomax distribution, Aust J Basic Appl Sci, 7(7) (2013b), 658-667.

[9] Bourguignon, M., Silva, R. B., Zea, L. M. and Cordeiro, G. M., The Kumaraswamy Pareto distribution, Journal of Statistical Theory and Applications, 12(2) (2013), 129-144.

[10] Choulakian, V. and Stephens, M. A., Goodness-of-fit tests for the generalized Pareto distribution, Technometrics, 43(4) (2001), 478-484.

[11] Elbatal, I., Asha, G. and Raja, V., Transmuted exponentiated Fréchet distribution: properties and applications, J. Stat. Appl. Prob, 3 (2014), 379-394.

[12] Elbatal, I. and Aryal, G., On the Transmuted Additive Weibull Distribution, Austrian Journal of Statistics, 42(2) (2016), 117-132.

[13] Eltehiwy, M. and Ashour, S., Transmuted exponentiated modified Weibull distribution, International Journal of Basic and Applied Sciences, 2(3) (2013), 258-269.

[14] Gentle, J. E., Random number generation and Monte Carlo methods, Springer Science \& Business Media, 2013.

[15] Gumbel, E.J., Bivariate Exponential Distributions, Journal of the American Statistical Association, 55(292) (1960), 698-707.

[16] Hussian, M. A., Transmuted exponentiated gamma distribution: A generalization of the exponentiated gamma probability distribution, Applied Mathematical Sciences, 8(27) (2014), 1297-1310.

[17] Johnson, N. L. and Kotz, S., A vector multivariate hazard rate, Journal of Multivariate Analysis, 5(1) (1975), 53-66.

[18] Lee, E. T. and Wang, J., Statistical methods for survival data analysis, John Wiley $\mathcal{E}_{3}$ Sons Vol. 476, 2003.

[19] Merovci, F., Transmuted Lindley distribution, International Journal of Open Problems in Computer Science and Mathematics, 6, (2013a).

[20] Merovci, F., Transmuted exponentiated exponential distribution, Mathematical Sciences and Applications E-Notes, 1(2) (2013b).

[21] Merovci, F. and Elbatal, I., Transmuted Lindley-geometric distribution and its applications, arXiv preprint arXiv:1309, 3774, (2013). 
[22] Merovci, F. and Puka, L., Transmuted pareto distribution, In ProbStat Forum, Vol. 7 (2014), $1-11$.

[23] Merovci, F., Transmuted Rayleigh distribution, Austrian Journal of Statistics, 42(1) (2016), $21-31$.

[24] Nasiru, S. and Luguterah, A., The new Weibull-Pareto distribution, Pakistan Journal of Statistics and Operation Research, 11(1), (2015).

[25] Rényi, A., On measures of entropy and information, In Proceedings of the fourth Berkeley symposium on mathematical statistics and probability, (Vol. 1) (1961), 547-561.

[26] Shaked, M. and Shanthikumar, J. G., Multivariate conditional hazard rate functions-an overview, Applied Stochastic Models in Business and Industry, 31(3) (2015), 285-296.

[27] Shaw, W. T. and Buckley, I. R., The alchemy of probability distributions: Beyond gramcharlier and cornish-fisher expansions, and skew-normal or kurtotic-normal distributions, Submitted, Feb, 7, 64, 2007.

[28] Shaw, W. T. and Buckley, I. R., The alchemy of probability distributions: beyond GramCharlier expansions, and a skew-kurtotic-normal distribution from a rank transmutation map, arXiv preprint arXiv:0901.0434, 2009.

[29] Singh, S. K., Singh, U., and Sharma, V. K., Estimation on System Reliability in Generalized Lindley Stress-Strength Model, J. Stat. Appl. Prob, 3, (2014), 61-75.

[30] Tahir, M. H., Cordeiro, G. M., Alzaatreh, A., Mansoor, M. and Zubair, M., A New WeibullPareto Distribution: Properties and Applications, Communications in Statistics-Simulation and Computation, 45(10) (2016), 3548-3567.

[31] Yilmaz, M., Hameldarbandi, M. and Kemaloglu, S. A., Exponential-modified discrete Lindley distribution, SpringerPlus, 5(1) (2016), 1660.

Current address: Monireh HAMELDARBANDI: Ankara University, Graduate School of Natural and Applied Sciences, 06110, Diskapi, Ankara, TURKEY.

E-mail address: monir6685@gmail.com

ORCID Address: http://orcid.org/0000-0002-3543-3709

Current address: Mehmet YILMAZ: Ankara University, Faculty of Science, Department of Statistics, Ankara, TURKEY.

E-mail address: mehmetyilmaz@ankara.edu.tr

ORCID Address: http://orcid.org/0000-0002-9762-6688 